\title{
Environnement et anthropisation dans le Pacifique, dégradations et mutations
}

Frédéric Angleviel

\section{OpenEdition}

12 Journals

Édition électronique

URL : https://journals.openedition.org/jso/5332

DOI : $10.4000 /$ jso.5332

ISSN : $1760-7256$

Éditeur

Société des océanistes

Édition imprimée

Date de publication : 15 décembre 2008

Pagination : 113-126

ISBN : 978-2-85430-012-3

ISSN : 0300-953x

Référence électronique

Frédéric Angleviel, «Environnement et anthropisation dans le Pacifique, dégradations et mutations », Journal de la Société des Océanistes [En ligne], 126-127 | Année 2008, mis en ligne le 15 décembre 2011, consulté le 21 septembre 2021. URL : http://journals.openedition.org/jso/5332 ; DOI : https:// doi.org/10.4000/jso.5332

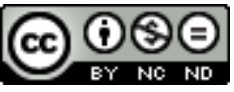

Journal de la société des océanistes est mis à disposition selon les termes de la Licence Creative Commons Attribution - Pas d'Utilisation Commerciale - Pas de Modification 4.0 International. 


\section{Environnement et anthropisation dans le Pacifique, dégradations et mutations}

par

Frédéric ANGLEVIEL*

\section{RÉSUMÉ}

À la suite du voyage de circumnavigation de Magellan (1521) débuta une période de "découverte mutuelle " entre les insulaires océaniens et les Européens. Cette période connut, à partir de 1788, une seconde vague de peuplement: beachcombers, traders, missionnaires puis fonctionnaires coloniaux et colons. On constate dès le XIX siècle l'accroissement de l'anthropisation des milieux (cultures commerciales, mécanisation) et une nette dégradation de l'environnement liée tant à l'irruption des espèces et des techniques occidentales (espèces intrusives, exploitations minières) qu'à la désorganisation des sociétés océaniennes traditionnelles. À l'aube du $X X I^{e}$ siècle, la dégradation des milieux et les mutations des sociétés océaniennes imposent que se mette en place une gestion de l'environnement afin d'offrir aux États océaniens, demain, des possibilités de survie et un développement durable.

MoTS-CLÉS : environnement, anthropisation, dégradation des milieux, mutations sociétales, Pacifique

\begin{abstract}
Following the circumnavigation travel of Magellan (1521) a mutual discovery intervened between native Oceanian peoples and Europeans. After 1788, the settlement comprised beachcombers, traders, missionaries, colonial officers and settlers. As soon as the XIX ${ }^{\text {th }}$ century the anthropization and the degradation of the environment (through the extension of commercial plantations and mecanization) became evident. This period was also that of the introduction of invasive species as well as that of the beginning disorganization of Oceanian societies. At the very begin of the $X X I^{\text {st }}$ century, the degradation of the environment and the increasing disorganization of the societies are such that an effective control and management of the environment must be organized. The future development and even, possibly, the survival itself of these societies are dependent on this decision
\end{abstract}

KEYwORDS: environment, anthropization, degradation of environments, societal mutations, Pacific area
L'exiguïté des îles du Pacifique, leur long isolement géographique ${ }^{1}$ et leur végétation endémi- que relativement peu diversifiée ont concouru à la fragilité des écosystèmes qu'ils abritaient.

1. Vu son isolement, l'archipel des Hawaii était peuplé de $99 \%$ d'espèces endémiques et, avant l'arrivée de l'homme, une nouvelle espèce arrivait par l'océan ou par les airs tous les 100000 ans. Aujourd'hui, vingt nouvelles espèces d'invertébrés arrivent chaque année, par le seul trafic aérien. Cet isolement général du Pacifique eut de nombreuses conséquences inattendues sur l'évolution des espèces. La quasi-absence de mammifères permit à des oiseaux ou des reptiles d'occuper toute la place disponible sur les îles : tortues géantes aux îles Galápagos, émeus géants (moa) en Nouvelle-Zélande. La quasi-absence d'herbivores explique que la plupart des espèces végétales développèrent peu de défenses « naturelles ». Les feux spontanés étant rares, les plantes furent particulièrement vulnérables aux techniques de brûlis des premiers arrivants. La quasi-absence de prédateurs permit aux premiers Océaniens de chasser jusqu'à l'extinction nombre d'espèces d'oiseaux coureurs : Moa en Nouvelle-Zélande, Sylviornis (un mégapode) en Nouvelle-Calédonie.

* Historien, fr.angleviel@canl.nc 
L'une des conséquences de cet isolement et de cet endémisme fut, par contre, une biodiversité remarquable, tant dans la flore que dans la faune. L'équilibre de ces deux composantes a été progressivement menacé par les activités humaines, historiques et contemporaines. Les ethnoarchéologues ayant présenté, dans plusieurs travaux, l'impact de la première vague de peuplement de l'Océanie insulaire (Dodson, 1992; Kirch et Hunt, 1997 ; McNeill, 2001), notre propos portera d'abord sur la seconde vague de peuplement qui, après la découverte mutuelle et les premiers contacts entre insulaires et découvreurs (1521-1840), vit la colonisation de quasiment toutes les îles du Pacifique (1840-1945). La troisième vague, depuis la fin de la Deuxième Guerre mondiale et la décolonisation politique qui s'ensuivit, porte sur des migrations constituées de plus en plus de cadres expatriés. Elle se perpétue aujourd'hui à travers la venue d'Asiatiques ou d'Occidentaux qui fournissent en même temps les crédits nécessaires aux États océaniens pour construire des routes (plan tourisme financé par les Américains au Vanuatu) ou bâtir des immeubles (parlement des Samoa financé par la Chine), qui fondent des usines de transformation de produits agricoles commercialisables ou de minerais et qui participent à l'abattage des forêts de la Mélanésie du Nord.

Nous allons donc présenter surtout l'impact des deux dernières vagues d'anthropisation des milieux naturels océaniens afin de définir s'il s'avère possible de mettre fin ou de minimiser la détérioration des milieux naturels. Pour ce faire, nous étudierons les pratiques d'utilisation des milieux exploitables, des plus anciennes aux plus modernes, ainsi que leurs incidences sur les rapports traditionnels des populations océaniennes avec leur environnement. Nous réfléchirons alors aux moyens permettant de s'orienter vers une meilleure gestion de l'environnement.

\section{Hier, ou la dégradation ponctuelle de l'environnement}

\section{Si l'on en croit Donald Garden :}

« a hybrid landscape, one with mixed indigenous and human-introduced species, is a degraded landscape because it represents the destruction of pre-existing ecosystems, often with the loss of species, and almost inevitably with a decline in biodiversity. » (2005 : XVI)

Cette dégradation est, de fait, inévitable puisque, depuis la première vague de peuplement de l'Océanie, l'homme croît et se multiplie. La deuxième vague de peuplement fut haute en péripéties mais peu destructrice. Les premiers contacts économiques et religieux entre Océaniens et Occidentaux (1788-1840) ont eu des impacts différents selon les modes et les lieux de ces actes de colonisation (1840-1945).

\section{La protohistoire fut une période de prédation}

La protohistoire recouvre la période durant laquelle les Océaniens ne possédaient pas l'écriture. Ceci implique que nos connaissances sur l'Océanie pendant cette période sont partielles et souvent partiales (Howe, 2000). Elles nous sont essentiellement provenues de la narration de legs oraux, des fouilles archéologiques et des récits des Occidentaux.

\section{- L'impact oublié des découvreurs}

Le temps de la découverte mutuelle, qui commence avec Magellan et se termine avec la création de la colonie pénitentiaire de Sydney en 1788, a eu sur la nature une incidence beaucoup plus profonde qu'on l'a longtemps pensé. En effet, si les historiens sont aujourd'hui convaincus de l'importance du choc microbien, qui entraîna le décès de $40 \%$ des habitants des grandes terres mélanésiennes et de $90 \%$ des Marquisiens, on oublie trop souvent que les découvreurs bouleversèrent de bien d'autres manières les fragiles écosystèmes du Pacifique (Bushnell, 1993). D'une part, la disparition accidentelle de près de la moitié de la population de l'Océanie entraîna un net recul de l'emprise humaine sur son environnement. La suppression ou la diminution de nombreux groupes humains implantés sur les rivages permirent aux autochtones refoulés autrefois dans les arrière-pays de revenir sur le bord de mer. C'est ainsi que les montagnes de Tahiti se dépeuplèrent au profit des plaines littorales. De même, les grands travaux agricoles comme les tarodières irriguées furent abandonnés et les friches se multiplièrent. Parfois, les forêts secondaires se développèrent et se rapprochèrent des villages océaniens. Le plus souvent, les herbivores importés par les Occidentaux profitèrent de l'abandon de nombreuses terres cultivées pour les coloniser et se multiplier. L'exemple contemporain des Galapagos, où l'introduction de trois chèvres en 1959 déboucha sur une population de 20000 caprins en 1971, montre quelle peut être la rapidité potentielle de l'introduction d'une espèce animale. Mais, d'autre part, l'introduction d'une nouvelle vague d'espèces végétales et animales eut des répercussions très importantes sur l'environnement. Tous les auteurs notent l'impact très négatif de l'introduction involontaire des rats norvégiens (Rattus norvegicus) et des rats noirs (Rattus rattus) tant 


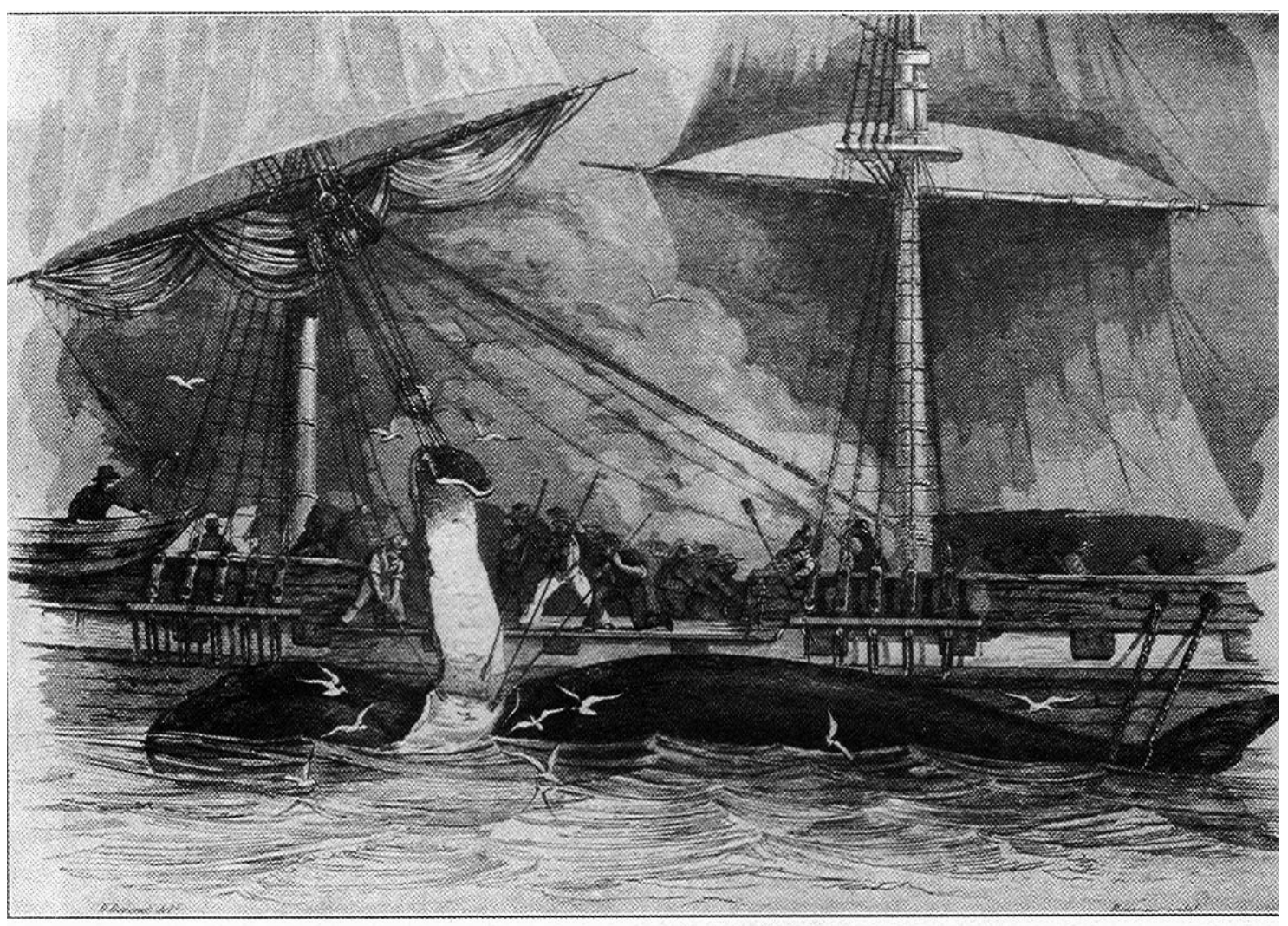

[78] “CUTTING IN" A WHALE.

From an engraving by Rouarque (about 1850) in the Peabody Museum, Salem.

FIGURE 1. - La chasse à la baleine : « Cutting in » a whale (gravure de Rouarque, circa 1850, Peobody Museum, Salem)

sur la flore que sur la faune ${ }^{2}$. Il en est de même pour le moustique qui fut introduit accidentellement dès 1826 aux îles Hawaii.

On sous-estime souvent l'impact particulièrement important des introductions volontaires de plantes agricoles ou d'animaux domestiques destinés à améliorer la vie des Océaniens et, secondairement, à préparer l'implantation ultérieure de colons. Quiros planta sans succès du maïs aux Marquises en 1595. James Cook offrit en 1774 au chef de Balade, dans le nord de la Grande Terre calédonienne, un couple de chiens puis un couple de porcs, sans se rendre compte que ces deux cadeaux entraîneraient une véritable catastrophe écologique. Les chiens retournèrent à l'état sauvage, se multiplièrent et détruisirent la faune endémique. Les cochons s'enfuirent dans la chaîne centrale et se rendirent responsables de l'érosion de sols et de la pollution des cours d'eau. Lapérouse fit réaliser par son équipage un jardin potager sur l'île de Pâques en 1786, espérant que les Pascuans pourraient à l'avenir diversifier leur régime alimentaire. Et que dire de l'introduction de la chèvre dans de nombreuses îles du Pacifique, quand l'on connaît la propension de cet animal à coloniser tous les milieux - sauf les forêts - non encore exploités par l'homme. L'absence de prédateur naturel impliqua le développement rapide et incontrôlé des troupeaux de caprins, capables de causer des dégâts réels et durables à l'environnement.

- Le temps des aventuriers accéléra l'invasion des espèces animales et végétales

Durant la période des premiers contacts, l'heure fut à la prédation. Les baleiniers américains et anglais détruisirent les derniers grands regroupements de baleines entre 1789 et 1860 .

2. C'est ainsi que les rats détruisent de nombreux œufs, voire des poussins. Lorsque le rat fut introduit dans l'île de Lord Howe en 1918, cinq espèces d'oiseaux disparurent. Lorsque ces rongeurs apprécient les graines ou les noix d'un arbre, voire ses jeunes pousses, celui-ci disparaît des forêts concernées. Il est considéré qu'un couple de rats peut, théoriquement, générer vingt millions de descendants en trois années. L'éradication du rat a été effective, bien qu'involontaire, dans une seule île, Eniwetak (Marshall). La première bombe à hydrogène y fut en effet testée en 1952. 
Entre 1810 et 1830 , ce sont plus de trois millions d'otaries qui furent décimées pour leurs peaux destinées à être principalement envoyées en Chine. À partir de 1820, ce furent les holothuries - ou bêches-de-mer - qui furent surpêchées. La surexploitation fut telle que ce commerce se raréfia de lui-même. La reconstitution des stocks à long terme permit la perpétuation de cette activité jusqu'aujourd'hui. Il est à noter que la raréfaction des holothuries dérangea peu les écosystèmes lagonaires alors que l'exploitation du bois de coupe nécessaire à leur conditionnement en vue de leur exportation, c'est-à-dire pour les ébouillanter puis les sécher, réduisit notamment les zones de forêt sèche aux Fiji.

Parallèlement, la première ruée sur le bois de santal (Santalum) toucha les archipels des Fiji (1804), Hawaii (1811) puis des Marquises (1814). La royauté hawaiienne bâtit sa puissance sur l'exploitation du santal. Lorsque la production s'effondra en 1829, le roi Kamehameha alla jusqu'à envoyer deux navires à la recherche de nouvelles racines de santal aux NouvellesHébrides. Ils disparurent à jamais. Après la disparition des gisements du Pacifique oriental, il fallut attendre 1840 et la découverte de santal à l'île des Pins pour qu'une deuxième ruée touche la Nouvelle-Calédonie, puis les NouvellesHébrides et, enfin, les îles Salomon (Shineberg, 1973). Ce bois odoriférant était exporté en direction de la Chine et l'absence de replantation entraîna sa raréfaction durable dans le Pacifique insulaire.

Les Océaniens participèrent à la mise en coupe réglée de ces ressources commercialisables difficilement reconstituables. Traditionnellement, les Maoris de l'île du Sud chassaient les baleines ou les otaries. Bientôt, les Maoris et de nombreux autres Océaniens fournirent jusqu'à la moitié des équipages des navires baleiniers. En 1850, 4000 Hawaiiens sillonnaient l'océan Pacifique sur des navires occidentaux (Bushnell, 1993). La collaboration active des Océaniens fut indispensable lors de la recherche, puis de la préparation, tant des holothuries que du bois de santal. Les Océaniens obtinrent dans ce but des outils métalliques (Shineberg, 1983 ; Leblic, 1988), des objets en verre, des produits de consommation (tissu, tabac, alcool) et secondairement des armes. Dans le même temps, ils abandonnèrent nombre de techniques de fabrication anciennes. Les hameçons en bois ou en nacre laissèrent ainsi la place aux hameçons métalliques. Le premier aventurier qui s'aventura à Wallis en 1828 pour le commerce des bêches-de-mer, le métis espagnolhawaiien, Manini, échangeait un hameçon contre un panier d'holothuries.
Certains parlent aujourd'hui de commerce inégal même si, d'une certaine façon, on pourrait dire que chacun y trouvait son compte! Les Océaniens recevaient des biens qu'ils ne pouvaient pas, dans un premier temps, créer ou reproduire ; les Occidentaux recevaient des produits à haute valeur ajoutée qu'ils revendaient, grâce à un commerce triangulaire organisé à partir de Sydney, sur le marché asiatique (Young, 2000). Il est patent que l'introduction des haches en fer, des récipients en verre ou des marmites en fonte modifia complètement les techniques artisanales de ces peuples qui eurent vite fait, par ailleurs, soit de s'approprier les techniques de fabrication de quelques-uns de ces biens, soit de récupérer les matières premières pour les intégrer dans leurs processus de fabrication traditionnels (voir par exemple Leblic, 1988).

Les populations océaniennes purent alors améliorer, par exemple grâce à de nouveaux outils, leurs techniques de défrichage et modifier leur environnement de manière plus rapide. Cette amélioration des méthodes culturales permit de contrebalancer la disparition d'une grande partie de la population active océanienne, les agriculteurs armés de coupe-coupe, de haches et de barres à mine, étant capables, même en nombre limité, de réaliser les travaux agricoles nécessaires à la survie des leurs (Raczy, 2005).

Les Océaniens entreprirent, afin d'acquérir ces biens hier inaccessibles, de développer leur emprise agricole afin de développer des cultures vivrières susceptibles d'être troquées ou vendues aux Occidentaux. Cette augmentation des terres cultivées à proximité des premières implantations européennes, impliqua non seulement le défrichement de nouvelles terres, mais aussi la propagation de nouvelles espèces, qui correspondaient au goût des nouveaux arrivants. C'est ainsi que, dès 1809, les Fijiens (de Mathuata) cultivaient des ananas ou des courges. Parallèlement, les Océaniens prenant goût au tabac, des plantations spontanées se développèrent auprès des ports et des lieux d'ancrage des navires occidentaux. C'est ainsi que les Futuniens prirent l'habitude de fumer dès les années 1830 leur propre tabac et, qu'aujourd'hui encore, ils cultivent artisanalement des plants de tabac malgré le monopole de l'État français en la matière. Les "rouleaux de tabac», paquets oblongs de feuilles séchées étroitement liées, furent longtemps un bien d'échange de haute valeur. Ils sont encore utilisés dans les îles isolées.

De manière plus planifiée, des Océaniens développèrent des entreprises agricoles à des fins d'exportation. C'est ainsi que les Tahitiens, sur 
les conseils de leurs pasteurs, réalisèrent dès 1793 des élevages de porcs destructeurs de l'environnement villageois. Cet élevage extensif permit d'envoyer des barils de porcs salés à destination des établissements pénitentiaires australiens. De même, dès la fin du XVIII ${ }^{\mathrm{e}}$ siècle, les Maoris de Nouvelle-Zélande cultivèrent des pommes de terre ${ }^{3}$, production agricole facilement transportable, à l'intention des mêmes administrations pénitentiaires australiennes.

\section{La colonisation généralisa les modifications envi- ronnementales}

Selon le mode de colonisation, l'impact de celle-ci fut plus ou moins important. Trois types de colonisation et trois types d'impact peuvent être distingués, selon que l'on se trouve dans les colonies de traite, celles de plantation ou celles de peuplement.

Dans les colonies de traite, comme à Wallis-etFutuna, à Tokelau ou à Tuvalu, c'est-à-dire dans les colonies où les Océaniens cultivaient euxmêmes des produits agricoles qu'ils troquaient à des trafiquants contre des produits de première nécessité, l'impact sur l'environnement a été mineur. En fait, la seule culture commerciale correspondant à la demande des Européens et à une production pré-européenne fut le cocotier. Les premiers arrivants l'avaient transporté avec eux dans toute l'immensité du Pacifique et ils en tiraient artisanalement de l'huile de coco.

L'Allemand Weber inventa en 1854 la technique de préparation du coprah, consistant à faire sécher la chair de coco afin de la presser ultérieurement dans des moulins. Cette technique permit de valoriser les cocoteraies existantes. De plus, les populations du littoral, généralement converties au christianisme, avaient besoin de ressources monétaires pour financer la construction et l'aménagement des temples et des églises. Aussi, les chefs, conseillés par les missionnaires, obligèrent-ils leurs sujets à planter des cocoteraies communautaires destinées essentiellement à financer les constructions religieuses, les écoles de mission et les premiers dispensaires. Le paysage littoral des îles hautes et tout l'écosystème des atolls en furent bouleversés. C'est ainsi que les missionnaires apprirent aux Océaniens à débrousser ces nouvelles cocoteraies et à les entretenir grâce à l'implantation de troupeaux de bovins ou de caprins. Les populations de rongeurs s'accrurent et la biodiversité régressa (Angleviel, 1990).

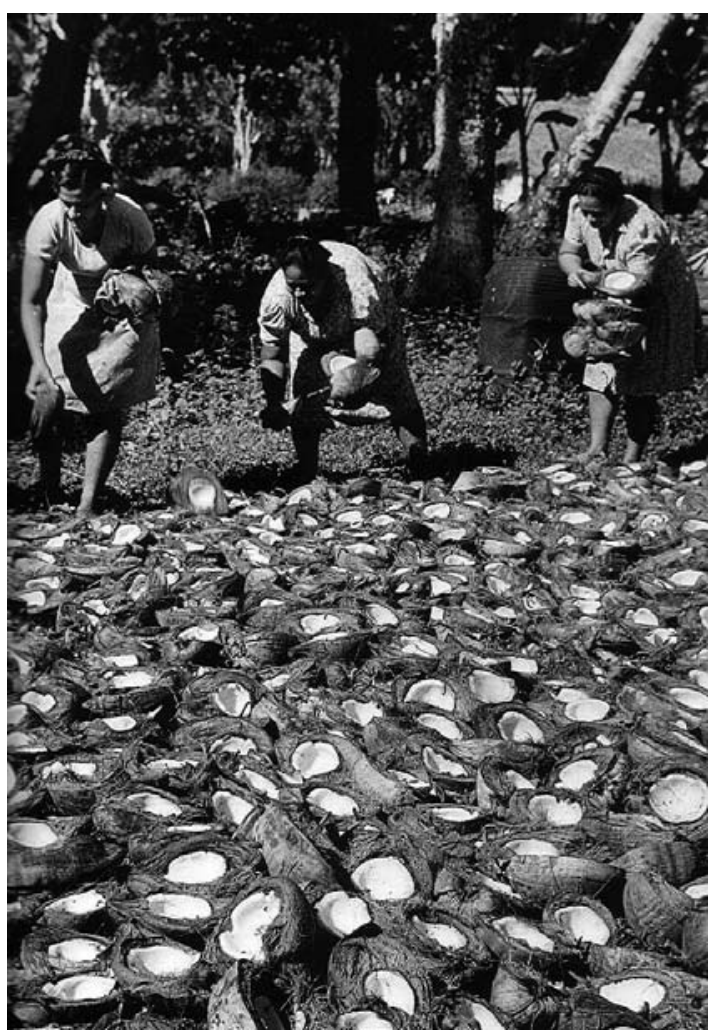

Figure 2. - Préparation du coprah aux Samoa (1920, coll. privée)

Dans les colonies de plantation, comme la Polynésie française, les Nouvelles-Hébrides ou les Samoa, les Occidentaux développèrent quelques cultures commerciales: des cocoteraies, dans toutes les îles, du coton aux Fiji ou aux Samoa, de la canne à sucre aux Fiji. Bien d'autres expériences d'implantation eurent lieu dans les îles du Pacifique, les résidents de France aux Nouvelles-Hébrides testant par exemple dans les années 1920 des cultures de café, de cacao, de poivre ou de ricin. Les planteurs pratiquèrent l'embauche de travailleurs engagés dans d'autres îles, considérés comme plus productifs et moins enclins à se révolter. L'engagement forcé ou blackbirding entraîna le départ de 100000 Mélanésiens vers le Queensland, dont un tiers seulement retournera ensuite dans leur archipel. Ainsi, 15000 Néo-Hébridais se rendirent en Nouvelle-Calédonie, $50 \%$ d'entre eux rentrèrent au bout des cinq années d'engagement (Shineberg, 2003).

Le développement de ces zones de monoproduction eut une incidence sur l'environnement : disparition d'espèces endémiques ou d'espèces introduites par les premiers Océaniens, diminu-

3. Elles furent introduites en Nouvelle-Zélande en 1770 par James Cook, en même temps que le porc, le blé et une graminée (canary grass). Les porcs, vaquant en liberté autour des villages maoris, causèrent de nombreux dégâts « collatéraux ». 


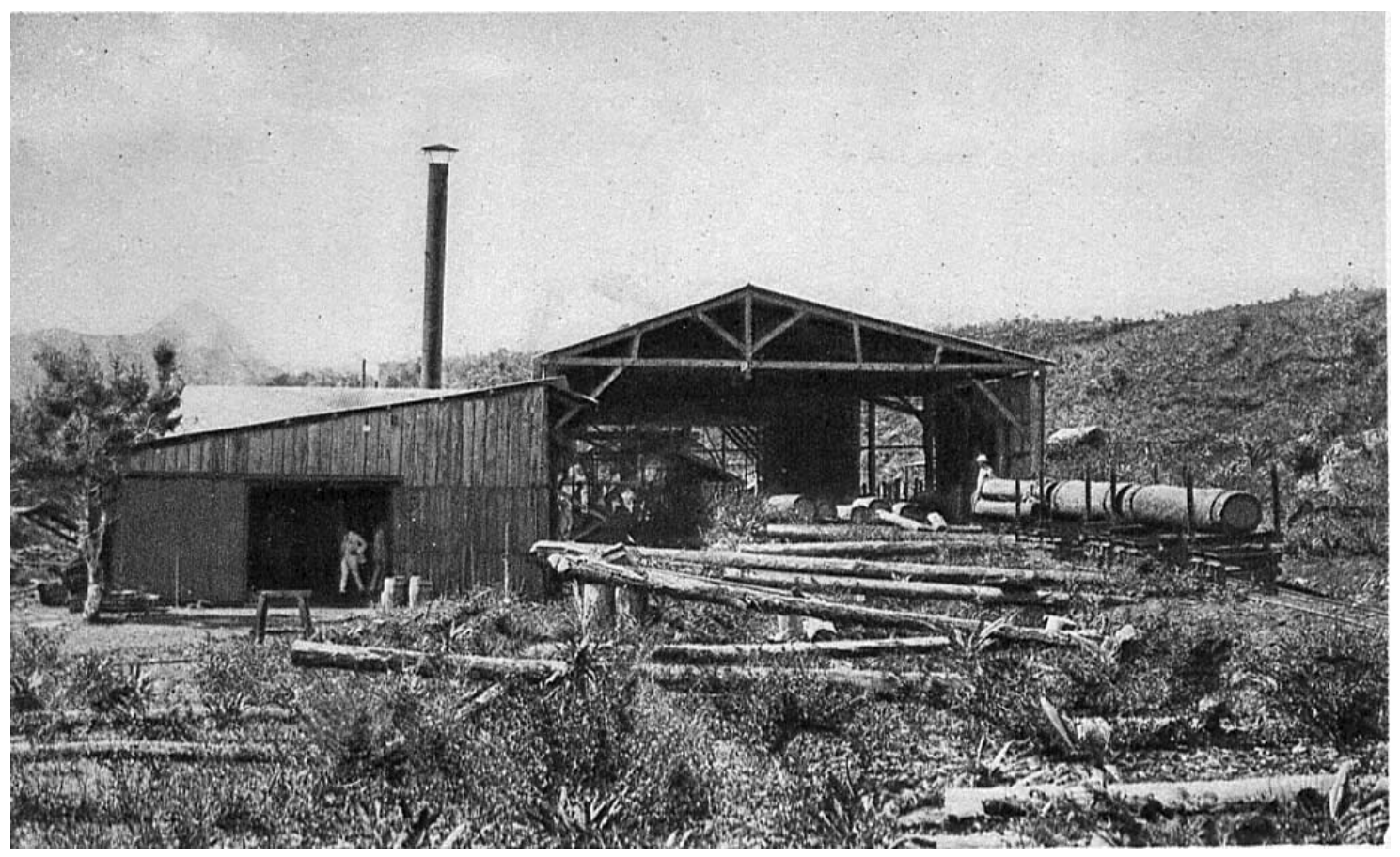

FIGURE 3. - Scierie mécanique dans la brousse calédonienne (carte postale Ballande, circa 1930)

tion progressive des ressources naturelles en eau, érosion et donc envasement des estuaires et des lagons, développement d'espèces parasites... Les rats se mirent ainsi à pulluler dans les champs de canne à sucre des Fiji et l'introduction de la mangouste indienne pour tenter de les éradiquer entraîna la disparition de sept espèces d'oiseaux endémiques. Les planteurs des NouvellesHébrides, après avoir planté des cocoteraies à tracés géométriques, les firent débroussailler et ensemencer en graminées par leurs employés ni-vanuatu afin d'y installer des troupeaux de charolais capables de maintenir à moindre frais les plantations en état tout en leur fournissant une ressource alimentaire supplémentaire.

Enfin, dans les colonies de peuplement, c'està-dire en Nouvelle-Calédonie, à Hawaii, en Nouvelle-Zélande et en Australie, les nouveaux arrivants ont fortement modifié les paysages indigènes car ils s'y sont installés en nombre et y ont reproduit les modes d'exploitation et de vie qu'ils connaissaient en Europe (Dunlap, 2000). Les administrations coloniales repoussèrent les Océaniens sur les marges pour installer des colons qui firent reculer les forêts et transformèrent les aires de culture grâce à l'usage de la charrue. Ils créèrent ainsi des paysages agricoles nouveaux : champs de blé ou de canne à sucre en Australie, pâturages à moutons en Nouvelle-
Zélande, champs de canne à sucre puis d'ananas à Hawaii (Ziegler, 2002). Les Océaniens, regroupés dans des régions excentrées et souvent montagneuses, durent solliciter fortement une nature moins généreuse, ce qui entraîna une réduction des temps de jachère et une augmentation des cultures sur pentes. Ceci développa l'érosion et mit en place un cercle vicieux qui fragilisa d'autant plus les sols et l'environnement.

Au fil du temps, l'introduction par les Occidentaux de nombreuses espèces comestibles devint ce que d'aucuns considèrent comme un bien. C'est ainsi que les habitants de la vallée d'Anahulu, sur l'île de Oahu (Hawaii), possédaient dès 1846, à côté des tubercules arrivés sur les pirogues de leurs ancêtres, du riz, du maïs, des haricots, de la salade, des melons, des concombres, du poivre, du café, du tabac, des oranges, des citrons ou encore des goyaves. L'ensemble des populations océaniennes bénéficia ainsi de cette diversification des ressources alimentaires.

Il est à noter que la colonisation de peuplement entraîna une modification radicale des rapports entre l'homme et la nature. Jusqu'alors, celle-ci dictait sa conduite à l'homme et les Pascuans subirent de terribles famines ${ }^{4}$ lorsqu'ils eurent fini de détruire les forêts de Rapanui, au $\mathrm{XVI}^{\mathrm{e}}$ siècle. L'homme avait désormais les moyens

4. Les Pascuans arrivèrent sans doute des Marquises vers les années 600 de notre ère. Parmi les espèces qu'ils introduisirent alors, l'arbre à pain et le chien avaient disparu avant l'arrivée des Européens. En l'absence de porcs (comme en Nouvelle- 
de transformer son environnement de manière profonde et de retarder durablement le prix écologique à payer. L'exemple le plus marquant de cette nouvelle échelle d'anthropisation est reconnu en Nouvelle-Zélande où les immenses forêts, improductives selon les critères occidentaux, ont été débitées afin de faire du bois d'œuvre et de permettre la création d'immenses pâturages à moutons. Les forêts, estimées à 18 millions d'hectares en 1840, ne couvrent plus aujourd'hui que 6 millions d'hectares alors que les pâturages sont passés de 8 à 14 millions d'hectares (McNeill, 2001). Il en est résulté une érosion très importante des sols, définitivement appauvris. Quant aux marais et autres zones inondables, leur surface a été réduite de $85 \%$, ce qui a entraîné, progressivement et discrètement, la disparition de nombreuses espèces (Taylor, 1997).

\section{Une transformation mineure mais généralisée du milieu}

Très naturellement, au travers de ces divers types de colonisation, les Occidentaux introduisirent en Océanie de nombreuses espèces, animales ou végétales. C'est ainsi qu'à Guam, première île océanienne à être colonisée dès 1668 par des missionnaires jésuites accompagnés de garnisons militaires, le maïs remplaça le riz cultivé par les Chamorros et que s'installa progressivement la culture du cacao, du café ou du tabac parallèlement au développement de l'élevage des chevaux, des cochons, des animaux de basse-cour, des bovins ou encore des buffles. Et, ceci, sans compter l'introduction des chiens, des chats, de nouvelles espèces de rats ou des cerfs, qui tous se répandirent dans l'île et modifièrent son fragile écosystème. Ici, comme cela se généralisera ensuite dans toutes les zones agricoles, les forêts furent brûlées afin de créer des pâturages et des nouvelles terres de culture. L'Espagne ayant perdu Guam au bénéfice des États-Unis en 1898, les Américains y implantèrent une base militaire dans la zone nord moins fertile puis développèrent des cocoteraies dans le Sud. L'occupation japonaise, entre 1941 et 1944, amena des destruc- tions inattendues et massives. Comme le rappelle D. Garden :

«The war destroyed large areas of jungle, reefs, beaches, and other habitat on Guam, and left tons of wrecked military equipment and garbage, which were bulldozed over cliffs into the ocean. » (2005: 139)

Parallèlement, la colonisation de l'Océanie réduisit les marges de manœuvre des peuples premiers qui furent souvent repoussés loin des côtes ou utilisés comme main-d'œuvre bon marché dans d'autres archipels. C'est ainsi que les Aborigènes d'Australie ou les Kanak de Nouvelle-Calédonie furent regroupés dans des réserves. La plupart des hommes valides des Mariannes furent déportés par les Espagnols vers Guam. Les Péruviens kidnappèrent des Gilbertins pour travailler dans leurs mines. Les Salomonais et les Néo-Hébridais furent engagés plus ou moins volontairement pour travailler dans les champs de canne à sucre de NouvelleGuinée et du Queensland.

Les Océaniens furent plus des spectateurs que des acteurs de la période coloniale. Les missions chrétiennes jouèrent un grand rôle dans leur renaissance culturelle et économique. En effet, les missionnaires protégeaient leurs fidèles autant qu'ils le pouvaient et ils leur apprirent des techniques utiles provenant de l'Occident, en commençant par l'écriture, la fabrication des planches de bois, la réalisation de vêtements ou encore l'utilisation des armes à feu... L'histoire de la mission mariste en Nouvelle-Calédonie montre que les missionnaires fournissaient, à titre individuel, aux catéchumènes kanak, des carabines et des munitions pour leur permettre de chasser des canards et d'autres oiseaux comestibles. Qui plus est, les pères maristes fournirent en 1856 des armes à feu aux Kanak des réductions de Saint-Louis et de La Conception lorsque les Kanak du Sud de la Grande Terre voulurent attaquer le front pionnier du Grand Nouméa.

À la veille de la Deuxième Guerre mondiale, il apparaît que ce que l'on appelle le milieu naturel, non seulement a été modifié une première fois par les premiers arrivants, mais a été transformé 
plus en profondeur lors de l'intégration de l'Océanie dans le monde connu des Occidentaux. De nouvelles espèces sont apparues, d'autres ont été exterminées, les zones directement intégrées à l'œkoumène ont fluctué et la déforestation a globalement progressé.

C'est ainsi que les paysages de Waikato, dans l'île du Nord de la Nouvelle-Zélande, sont entièrement artificiels : les forêts primaires ont été abattues, les herbacées sont originaires d'Angleterre, les arbres replantés pour protéger les zones les plus érodées sont des peupliers de Lombardie ou des conifères d'Asie et les truites des rivières proviennent d'Europe (Pawson et Brooking, 2002).

Toujours en Nouvelle-Zélande, les nouveaux arrivants, regrettant l'inexistence d'herbivores sauvages susceptibles d'être un complément carné tout en procurant aux grands propriétaires terriens les joies de la chasse, créèrent de nombreuses sociétés d'acclimatation (Brennan, 2004). L'importation de différentes espèces de cerfs a débouché aujourd'hui sur une activité d'élevage importante, mais que dire de l'importation de chamois, d'élans, de gnous, d'antilopes (île Kawau) ou encore de lamas! Quelques renards furent même importés pour la chasse à courre, mais ils disparurent rapidement. $A$ contrario, l'opossum d'Australie, importé pour sa fourrure, et le lapin européen importé pour sa chair ont réussi dès les années 1830 leur acclimatation en Nouvelle-Zélande, où ils menacent les espèces autochtones par leur opportunisme. Les populations de lapins ont diminué après l'introduction illégale de calicivirus (virus induisant une maladie hémorragique interne du lapin) en provenance d'Australie, en $1997^{5}$.

\section{Aujourd'hui, une dégradation généralisée de l'environnement}

Depuis la Deuxième Guerre mondiale, qui fut marquée par une surpopulation très temporaire de la plupart des milieux insulaires, la démographie des États océaniens explose et les atteintes inévitables au milieu s'accentuent. Certaines auront des incidences sans gravité sur le milieu alors que d'autres semblent préoccupantes.

\section{L'accélération des dégradations}

Longtemps, les Océaniens furent peu nombreux et mal outillés. L'explosion démographique, l'arrivée de nouvelles populations et l'introduction de nouvelles techniques, permirent à l'homme de marquer toujours plus son empreinte sur la nature et d'y laisser des marques de dégradation.

- Des dégradations physiques liées à l'augmentation de la population

Tout accroissement de la population entraîne la nécessité de défricher de nouvelles terres et donc d'étendre le domaine des zones anthropiques considérées par les environnementalistes comme des zones dégradées. Seules les ex-colonies de peuplement, du fait de la mise en valeur intensive de certaines zones agricoles et de l'importation de ressources alimentaires, arrivent à se suffire, sans pour autant élargir exagérément les zones d'exploitation.

Il apparait que les migrations de travailleurs hors des îles du Pacifique eurent pour effet de réduire temporairement la pression démographique et donc la surexploitation de l'environnement. C'est ainsi que l'île de Futuna envoie depuis les années 1950 la plupart de ses enfants travailler en Nouvelle-Calédonie. Néanmoins, en raison de la croissance continue de la population, les Futuniens ont entrepris progressivement de réduire les temps de jachère et de cultiver des terrains sur des pentes de plus en plus fortes. La productivité diminue et l'érosion progresse.

A contrario, l'introduction de travailleurs immigrés en Océanie a entraîné une surexploitation des terres du bord de mer et l'introduction de pratiques agricoles souvent peu adaptées aux réalités de l'Océanie. Les Indiens venus aux Fiji y importèrent en 1873 la mangouste déjà citée. Les Japonais amenèrent dans tous les territoires micronésiens qu'ils occupèrent pendant l'entredeux-guerres le trop fameux escargot géant d'Afrique (Achatina fulica). Cet escargot est désormais présent en Nouvelle-Calédonie, à Wallis-et-Futuna, au Vanuatu, à Tahiti et aux Samoa. Une colonie fut découverte en Australie, à Cairns, en 1997 et semble avoir été éradiquée. Les Australiens tentent depuis 2004 d'empêcher l'implantation de ce gastéropode dans le

5. Les lapins furent longtemps un fléau en Australie, les barrières anti-lapins s'avérant inefficaces. Aussi, le gouvernement introduisit en 1950 la myxomatose le long de la rivière Murray. L'épidémie entraîna la quasi-disparition de l'espèce. Sa population se stabilisa bientôt à $5 \%$ du nombre préexistant dans les zones humides et à $25 \%$ dans les zones arides. Comme ce nombre était toujours important, de nouvelles recherches furent menées en 1995, sur une petite île d'Australie du Sud, sur le calicivirus, maladie hémorragique du lapin. Suite à une introduction illégale sur le continent, $90 \%$ des lapins décédèrent. Autant le développement des lapins avait porté atteinte à certaines espèces indigènes, autant sa quasi-disparition pose d'autres problèmes. Les prédateurs (oiseaux de proie, renards, chats) ne pouvant plus intégrer cette contribution à leur alimentation font peser une pression accrue sur les populations de marsupiaux et d'oiseaux autochtones (Coman, 1999). 
Queensland. En effet, dans les îles où il s'est implanté, il a entraîné la quasi-disparition des espèces autochtones d'escargots et s'est attaqué à la quasi totalité des espèces végétales comestibles.

Comme cela parait logique, l'introduction volontaire d'espèces végétales ou animales se poursuit. La Nouvelle-Zélande compte désormais, à côté des 2700 espèces végétales indigènes, 20000 espèces introduites, dont 200 sont considérées comme des espèces nuisibles. À Hawaii, il y aurait 5000 espèces végétales introduites. McNeill considère qu'elles ont créé « $a$ pantropical biota, with plants from India, China, Australia and the Americas, as well as some temperate invaders » (McNeill, 2001 : 317). En Australie, 2850 espèces végétales ont quitté les jardins urbains pour intégrer le paysage. Chaque année, une quinzaine de nouvelles espèces intrusives serait décomptée. Aujourd'hui, le Prickly Acacia infeste sept millions d'hectares alors que le lantana dénaturerait quatre autres millions d'hectares. Il est estimé que le surcoût annuel pour limiter la prolifération de ce type d'acacias s'élèverait, pour l'Australie, à quatre milliards de dollars.

Pour la faune, le gouvernement australien introduisit en 1935 dans le Queensland le crapaud de la canne à sucre (Bufo marinus) originaire de l'Amérique centrale. Non seulement celui-ci ne détruisit pas le cancrelat de la canne, qu'il était censé contenir, mais il fit disparaître de nombreuses espèces, dont des amphibiens et des petits mammifères (Low, 1999). De même, lorsque les Américains voulurent éradiquer d'Hawaii l'escargot géant d'Afrique, ils introduisirent un escargot américain (Euglandina rosea) mangeur d'escargots. Le prédateur ne s'attaqua pas au formidable escargot africain, mais élimina vingt espèces d'escargots autochtones. Mc Neill considère que l'extinction involontaire d'une centaine d'espèces endémiques est due à l'introduction volontaire d'espèces allogènes intrusives prédatrices.

Bien sûr, nombre d'espèces n'arrivèrent pas à s'implanter durablement, ce qui réduisit leur incidence sur les différents milieux. C'est ainsi que dès 1865 l'ingénieur agronome Boutan importa en Nouvelle-Calédonie des kangourous ou des cygnes noirs d'Australie. Aucun d'eux n'a eu de postérité. Certaines espèces importées pour des raisons ornementales sont devenues de véritables fléaux comme le myconia en Polynésie française (Gabrie, 1995).

Par ailleurs, l'introduction involontaire d'espèces intrusives s'accroît et certaines s'avèrent menaçantes (Quammen, 1996). C'est ainsi que l'introduction accidentelle à Guam, durant la Deuxième Guerre mondiale, du serpent brun de Mélanésie du Nord (Boisa irregularis) entraîna la disparition de quasiment tous les oiseaux de l'île (Jaffe, 1994). On oublie souvent les espèces marines. Or, le développement des liaisons maritimes et la pratique consistant à emplir ou à vider en mer les ballasts des bateaux ont permis la dispersion de nombreuses espèces océaniques. L'étoile de mer du Pacifique nord s'est répandue le long des côtes australiennes. En 1999, les Australiens ont dû mettre en place un programme onéreux pour éradiquer la moule noire (Black Striped Mussel) du port de Darwin.

Paradoxalement, l'anthropisation a aussi entraîné un fort développement de certaines espèces autochtones qui ont réussi à s'adapter aux nouvelles conditions créées par l'urbanisation (Low, 2002). En Australie, les mouettes argentées envahissent les villes côtières alors que les opossums colonisent les toitures et les parcs. De même, la nature étant opportuniste, de nombreuses espèces végétales se sont développées hors de leur niche écologique originelle, grâce au développement des jardins et aux déplacements humains. Généralement, cette nouvelle forme d'hybridation des paysages entraîne la disparition d'espèces autochtones.

- Des activités d'exploitation minières hautement destructrices

Les minerais sont exclusivement présents dans les régions originaires du continent géologique originel, c'est-à-dire l'Australie, l'arc mélanésien et la Nouvelle-Zélande. Ceci explique l'exploitation d'or et de cuivre en Nouvelle-Guinée, la production temporaire de manganèse dans l'île de Vaté (Forari) aux Nouvelles-Hébrides et celle de nickel, de chrome, de cobalt ou de cuivre en Nouvelle-Calédonie. Certaines activités minières commencèrent dès le $\mathrm{XIX}^{\mathrm{e}}$ siècle, comme l'exploitation du nickel en Nouvelle-Calédonie dès 1876, mais elles s'accrurent avec la mécanisation à partir des années 1960 .

A contrario, les gisements de guano, composés de déjections d'oiseaux de mer transformées par le temps en phosphate, furent exploités dès le début $\mathrm{du} \mathrm{Xx}^{\mathrm{e}}$ siècle. Le phosphate fut exploité à Nauru, dans l'île Banaba aux Kiribati et à Makatea en Polynésie française (Newbury, 1972). Paradoxalement, cette richesse exportable entraîna l'exode forcé des Banabans et une perturbation durable du mode de vie des Nauruans. Cette île, tout d'abord très riche, a frôlé au début des années 2000 la cessation de paiement qu'elle a évité en recevant des camps de transit australiens où étaient regroupés des boat people pakistanais, afghans et indonésiens. Aujourd'hui, une 
nouvelle phase d'exploitation du phosphate nauruan est en cours, qui finira de faire de cette île un récif fossile et dénudé.

Dans tous les cas, les exploitations minières autorisées par les États océaniens s'avèrent particulièrement destructrices au niveau environnemental, qu'il s'agisse de la destruction du couvert végétal, de l'extraction à ciel ouvert des minerais, de l'abandon des terres stériles sur les bas-côtés, de la pollution des rivières proches, de la mort des récifs frangeants (Thio en NouvelleCalédonie) ou des rejets des usines de première transformation (Pelletier, 2003). Ces questions sont actuellement à l'ordre du jour en NouvelleCalédonie (Capecchi et al., 2006), qu'il s'agisse d'accepter ou de refuser les rejets dans l'océan de l'extraction du manganèse (projet minier de Goro) ou de limiter l'émission des fumées polluantes de l'usine de Doniambo (sLn, Nouméa).

Dans les États océaniens pauvres, la question n'est pas la protection de l'environnement mais la répartition des revenus de la mine. L'affaire de Bougainville (au nord de la Papouasie NouvelleGuinée), partie entre autres de la question de la pollution des rivières par la plus grande mine de cuivre du monde, dériva rapidement sur le problème d'une répartition éventuelle de royalties entre les tribus minières ou l'ensemble de la population de l'île de Bougainville. Finalement, la mine de Panguna ferma ses portes en 1989. Aujourd'hui, le niveau économique de l'île de Bougainville s'est effondré et nombreux sont ses habitants qui espèrent la réouverture de la mine, quelles que soient les contraintes environnementales qu'elle pourrait engendrer.

\section{De nouvelles atteintes à l'environnement}

L'Océanie est de moins en moins éloignée du reste du monde et l'évolution des techniques permet son intégration pleine et entière dans le concert des nations. Aussi, l'anthropisation s'y développe-t-elle, avec ses dégradations environnementales (Mokaddem, 2003).

\section{- Zones de dégradation dans l'aire Pacifique}

L'océan Pacifique a été utilisé à partir de la Guerre du Pacifique et jusqu'en 1996 comme champ d'essai d'armes nucléaires, tant par les Américains (1946-1958 : Bikini aux îles Marshall, Christmas Island aux îles Kiribati), les Anglais (1952-1958 : Maralinga en Australie et 1957, à Christmas Island aux îles Kiribati) que les Français (1966-1996: Mururoa et Fangataufa). À ce jour, les États-Unis continuent d'utiliser le territoire océanien pour tester leurs missiles intercontinentaux non armés.
Dans tous les cas, il est certain que les essais nucléaires, en particulier les expérimentations dans l'atmosphère, eurent des répercussions négatives sur l'environnement marin et terrestre qui restent encore à évaluer. C'est ainsi que l'atoll de Bikini, quoi qu'en disent les Américains, reste à ce jour inhabitable et l'accès à Mururoa et Fangataufa est interdit aux populations.

En ce qui concerne la faune de l'océan Pacifique, elle fut quasiment préservée jusque dans les années 1960, les Océaniens ne possédant que des techniques de pêche lagonaire et les Occidentaux, longtemps incapables de préserver les poissons sur de longues durées, ont uniquement chassé les baleines pour leur huile. La révolution de la réfrigération mobile, la généralisation de l'usage des boîtes de conserve et la mondialisation de l'alimentation, entraînèrent l'apparition de chalutiers comportant des caissons réfrigérés puis la création de flottilles de pêche accompagnées par un navire-usine capable de préparer, voire de transformer, les produits de la pêche en haute mer.

La surpêche est évidente, en particulier pour les thonidés (marlin, bluefin tuna), du fait de l'utilisation systématique des filets tournants et des filets dérivants. Les États océaniens pourraient s'y opposer sur le plan formel mais, en l'absence de moyens de contrôle efficaces, ils préfèrent accueillir les flottilles pendant leurs relâches et obtenir le renouvellement, année après année, des droits de pêche payés par les pays «pêcheurs", États-Unis et Japon. Ces taxes représentent $45 \%$ des recettes de l'État de Kiribati et $25 \%$ des revenus des îles Marshall.

À une échelle plus générale encore, les écosystèmes marins de l'océan Pacifique portent la marque de l'évolution atmosphérique globale. Pour D. Garden (2005), les réchauffements périodiques de l'océan Pacifique, sous le contrôle des processus El Nino entrainent la mort d'une grande partie des coraux, car l'algue symbiotique qui leur donne leurs couleurs nécessite une température comprise entre $21^{\circ} \mathrm{C}$ et $28^{\circ} \mathrm{C}$. Lors d'une période de réchauffement en 1998 , c'est $20 \%$ du récif mondial, dont $3 \%$ de la Grande Barrière de récif australienne qui a disparu. La mort des récifs durant le $\mathrm{XXI}^{\mathrm{e}}$ siècle entraînerait une chute drastique des ressources maritimes côtières et rendrait la plupart des atolls très sensibles à l'érosion et aux cyclones (Dupon, 1985).

- L'impact d'une urbanisation rapide et souvent spontanée

Parmi les principaux problèmes que pose la dégradation de l'environnement, on note, à 
l'aube du $\mathrm{xxI}^{\mathrm{e}}$ siècle, la gestion des ensembles urbains. Le développement des populations océaniennes et leur concentration dans les villes vont croissant, ce qui pose le problème de l'extension des surfaces de logement, de la gestion des déchets, de l'insuffisance chronique des systèmes de tout-à-l'égout, de la pollution des eaux et, partant, des atteintes aux littoraux.

Les gouvernements des États océaniens créés après la Deuxième Guerre mondiale n'ont ni les moyens humains ni les moyens financiers de contrôler le développement urbain, qu'il s'agisse des règles d'urbanisation ou, simplement, de l'extension des habitats spontanés. Ces paysages de cabanes ou de squats, partout présents à partir des années 1980, ont été acceptés par la première génération de migrants, habitués à des conditions de vie rurales spartiates. Il devient indispensable de trouver des solutions pour la deuxième génération, non seulement pour éviter d'éventuelles explosions sociales, mais aussi pour résorber, ou tout du moins pour stabiliser, les phénomènes de péri-urbanisation spontanée. Ceux-ci échappent, en effet, à toute opération de gestion de l'environnement (Lobban et Schefter, 1997).

Comme partout dans le monde, la question énergétique a des répercussions sur les environnements océaniens. Les formes fossiles d'énergie (charbons, pétroles, gaz) y sont quasiment toutes importées. Leur utilisation progresse essentiellement en Australie, qui a refusé de signer en 1997 le traité de Kyoto portant sur la réduction de l'émission des gaz à effets de serre. En effet, alors que le traité demande aux États de réduire de $5 \%$ leurs émissions de dioxyde de carbone avant 2012, l'Australie considère qu'elle devra les augmenter de $8 \%$ dans le même temps pour maintenir son niveau de vie et d'activités économiques. Une des solutions à ce dilemme sera donc l'utilisation, dans l'avenir, des biocarburants comme cela se pratique de plus en plus en Europe (leur combustion produit autant de $\mathrm{CO}_{2}$ que le pétrole. La différence avec le pétrole est que l'on fabrique ses biocarburants chez soi). Le CIRAD a développé dans son centre de Santo au Vanuatu un biocarburant à base d'huile de coco qui fonctionne très bien, tant que la température ne descend pas au-dessous de $22^{\circ}$. En NouvelleZélande, la construction de barrages hydroélectriques dans les années 1960 fut freinée par les environnementalistes, qui eurent globalement gain de cause lors de l'arrivée au pouvoir des travaillistes en 1972.

Au niveau des nouvelles activités économiques tertiaires liées à la concentration de la vie dans les villes, le tourisme génère un effet indirect important sur les écosystèmes, tant par la construction d'hôtels que par l'épandage des pesticides sur les terrains de golf. Il participe aussi à une plus grande pression sur les milieux dit naturels puisque les touristes recherchent les zones les plus authentiques et les plus préservées. Ce faisant, le tourisme pousse à la création de nouvelles routes, au développement de la desserte maritime des îlots peu habités ou à l'implantation d'aérodromes dans les atolls les plus éloignés. Et, de fait, se pose la question du nécessaire équilibre entre les aspirations du mouvement écologique, qui souhaite limiter toute atteinte à l'intégrité de la nature, et celles de l'écotourisme, pour lequel la nature est un véritable produit économique, dont la préservation est indispensable au développement durable de la plupart des États du Pacifique insulaire. La Nouvelle-Zélande semble avoir trouvé à ce dilemme un compromis acceptable. Sa première réserve paysagiste, destinée à préserver intact l'environnement des geysers de la région de Rotorua, date de 1885 . La protection de la nature à des fins économiques y fut généralisée en 1903 par le scenery preservation act.

La destruction des forêts primaires de Mélanésie destinée à fournir l'Asie du Sud-Est en bois exotique pour la fabrication de meubles à destination des pays occidentaux, se trouve confrontée à un véritable problème régional : l'absence d'opérations de reboisement y entraîne, en effet, une érosion intense des sols dénudés sous l'effet des pluies tropicales.

En ce début de $\mathrm{XXI}^{\mathrm{e}}$ siècle, il apparaît que les dégradations régionales dues à l'anthropisation restent limitées dans le Pacifique (Tong et Cox, 2000), à l'exception de celles qu'a induites l'exploitation excessive des forêts et des sites miniers de Mélanésie. Ceci s'explique par le développement tardif de la cinquième région du monde et par l'existence de la faible pression démographique qui y règne ${ }^{6}$. Au vu de l'accélération des dégradations, il apparaît que des poli-

6. Donald Garden considère que la principale raison de la crise mondiale de l'environnement provient de l'expansion occidentale depuis près de 250 ans. Pour lui, trois problèmes l'emportent: "climate change and global warming; hybrid landscapes and blended biotas; and land clearance and soil degradation. But, to finish on a positive note, it must be recognized that at the international, national, and local levels, a great deal of attention has now turned toward finding solutions to our enormous environmental problems. » (2005 : 183). 
tiques régionales sont à mettre en œuvre ${ }^{7}$. Le Programme régional océanien pour l'Environnement (PROE) a pour mission de sensibiliser les gouvernements du Pacifique Sud à cette nécessité. Il serait souhaitable que le PROE initie et facilite des programmes de recherches scientifiques en sciences de la nature et en sciences humaines et sociales afin de mieux comprendre les interactions entre l'homme et la nature, sachant que celle-ci n'est plus naturelle depuis l'arrivée des premiers Aborigènes d'Australie il y a près de 60000 ans et que l'action de l'homme y a été, depuis, multiforme.

Par ailleurs, il est acquis que les modifications climatiques globales liées aux activités humaines (Philander, 1998) ont un impact sur l'Australasie. Certains considèrent que le réchauffement climatique va entraîner à moyen terme la submersion de Tuvalu ${ }^{8}$. D'autres considèrent que la réduction de la couverture forestière australienne induit une réduction des pluies et donc une augmentation de la salinité dans les régions céréalières de l'Australie de l'Ouest. Enfin, sur le plan climatologique au niveau mondial, l'alternance de El Niño et La Niña est responsable de multiples manifestations géodynamiques telles que l'érosion des sols et la sécheresse en Australie ou des cyclones accompagnés de fortes précipitations en Polynésie. Des études en sciences exactes replacées dans le cadre mondial, comme l'article consacré dans ce même numéro à l'analyse de l'histoire climatique du Pacifique sont donc, elles aussi, nécessaires pour que nous soyons à même de distinguer des effets physiques indépendants des activités humaines et des effets où la responsabilité de l'homme est pleinement engagée.

\section{BIBLIOGRAPHIE ${ }^{9}$}

Angleviel Frédéric, 1990. Faune et flore de NouvelleCalédonie, Nouméa, CTRDP (Centre territorial de recherche et de documentation pédagogique, collection Fenêtre sur... 1.

Brennan Claire, 2004, Imperial game: A History of Exotic Species, Hunting Society and Environment in New Zealand and Victoria, 1840-1901, Ph.D, University of Melbourne.

Bushnell O., 1993. The Gifts of Civilization: Germs and Genocide in Hawai'i, Honolulu, University of Hawai'i Press.

CAPECCHI Bernard et al., 2006. 101 mots pour comprendre l'environnement de la Nouvelle-Calédonie, Nouméa, éditions Île de Lumière, collection 101 mots pour comprendre la Nouvelle-Calédonie 8.

Coman Brian, 1999. Tooth and Nail: the story of the Rabbit in Australia, lieu, Text publishing.

Crossby Alfred, 1986. Ecological Imperialism: The Biological Expansion of Europe, 900-1900, Cambridge, Cambridge University Press.

DoDson John (ed.), 1992. The Native Lands: Prehistory and Environmental Change in Australia and the Southwest Pacific, Londres, Longman.

Dunlap Thomas, 2000. Nature and the English Diaspora: Environment and History in the United States, Canada, Australia and New Zealand, Cambridge, Cambridge University Press.

Dupon Jean François, 1985. Préparation aux désastres et expériences des désastres en Nouvelle-Calédonie, Honolulu, East West Center, PIDP.

FlanNery Tim, 1994. The Future Eaters. An ecological History of the Australian Lands and People, London, Reed Books.

GABRIE Catherine, 1995. L'état de l'environnement dans les territoires français du Pacifique Sud, Paris, ministère de l'Environnement, Institut français de l'Environnement.

GARDEN Donald, 2005. Australia, New Zealand and the Pacific. An environmental history, Sydney, ABC Clio.

Howe Kerry (ed.), 2000. Nature, Culture and History: the knowing of Oceania, Honolulu, University of Hawaii Press.

JAFFe Mark, 1994. And No Birds Sing: The Story of an Ecological Disaster in a Tropical Paradise (Guam), New York, Simon and Schuster.

KIRCH Patrick and Terry Hunt (eds), 1997. Historical Ecology in the Pacific Islands: Prehistorical Environmental and Landscape Change, Yale, Yale University Press.

LEBLIC Isabelle, 1988. L'évolution des techniques de pêche en Nouvelle-Calédonie, Techniques \& Culture 12, pp. 81-119.

7. Donald Garden considère aussi que : "The achievement of sustainability will require revolutionary changes in the way we think, the way we live, the way we deal with the environment. A change in mentality and ethics is required so that we see ourselves as part of the environment, not apart from it [...] We also need to recognize that it is in the interests of our own species as much as all others that we should treat the environment better [...]. Once it is realized that there is no economy without an environment, that there is no profit in a devastated world, attention will turn seriously to protecting what is left-and making a profit out of it. » (2005:208).

8. Soit neuf atolls dont la hauteur n'excède pas cinq mètres. Les 11000 habitants de cet État pourraient devenir des « sans domicile fixe ». Leur gouvernement a demandé la possibilité éventuelle d'une relocalisation générale : si la réponse de l'Australie est négative, la Nouvelle-Zélande a donné une réponse positive.

9. Quasiment toutes les études reposant en Océanie sur la signification des termes « environnement » et « anthropisation », sont en langue anglaise. 
LobBan Christopher and Maria Schefter, 1997. Tropical Pacific Island Environments, Agana, University of Guam Press.

Low Tim, 1999. The Untold History of Australia Exotic Invaders, Sydney, Viking.

_, 2002. The New Nature: Winners and Losers in Wild Australia, Melbourne, Penguin.

McNeILl J.R., 2001. Of Rats and Men: A synoptic Environmental History of the Island Pacific, in J.R. McNeill, Environmental History in the Pacific World, Londres, Ashgate Publishing.

MoKaddem Hamid (éd.), 2003. Approches autour de culture et nature dans le Pacifique Sud, Nouméa, Corail-éd. Expression.

Newbury Colin, 1972, The Makatea Phosphate concession, in Gerard Ward, Man in the Pacific Islands: Essays on Geographical Change in Pacific Islands, Clarendon, Clarendon University Press, pp. 1-90.

PAwson Eric and Tom Brooking (eds), 2002. Environmental Histories of New Zealand, Dunedin, Otago University Press.

Pelletier Bernard, 2003. Mines et environnement, in H. Mokaddem (éd.), Approches autour de culture et nature dans le Pacifique Sud, Nouméa, Corail-éd. Expression, pp. 195-213.

PhILANDER George, 1998. Is the Temperature Rising? The Uncertain Science of Global Warming, Princeton, Princeton University Press.
QuAmmen David, 1996. The song of the Dodo: Island Biogeography in an age of Extinctions, New York, Scribner.

RACZY Damien, 2005. Recul des tarodières irriguées en pays kanak, outils en fer et progrès technique, DEA Sociétés et cultures dans le Pacifique insulaire, université de la Nouvelle-Calédonie.

SHINEBERG Dorothy, 1973. Ils étaient venus chercher $d u$ santal, Nouméa, Société d'études historiques de la Nouvelle-Calédonie, publication de la Société d'études historiques de la Nouvelle-Calédonie 3.

—, 2003. La main-d'œuvre néo-hébridaise en NouvelleCalédonie, 1865-1930, Nouméa, Société d'études historiques de la Nouvelle-Calédonie, publication de la Société d'études historiques de la NouvelleCalédonie 61.

Tong Richard and Geoffrey Cox, 2000. Clean and Green. The New Zealand Environment, Auckland, David Bateman.

Young Ann, 2000. Environmental Change in Australia since 1788, Oxford, Oxford University Press.

ZIEGLER Alan, 2002. Hawaian Natural History, Ecology and Evolution, Honolulu, University of Hawaii Press. 
